\title{
Redox-sensitive Pluronic FI27-tocopherol micelles: synthesis, characterization, and cytotoxicity evaluation
}

This article was published in the following Dove Press journal:

International Journal of Nanomedicine

3 April 2017

Number of times this article has been viewed

Yuling Liu ${ }^{1,2, *}$

Sai $\mathrm{Fu}^{1, *}$

Longfei Lin'

Yuhong $\mathrm{CaO}^{3}$

$\mathrm{Xi} \mathrm{Xie}^{3}$

Hua $\mathrm{Yu}^{2}$

Meiwan Chen²

Hui $\mathrm{Li}^{\prime}$

'Institute of Chinese Materia Medica, China Academy of Chinese Medical Sciences, Beijing, ${ }^{2}$ State Key Laboratory of Quality Research in Chinese Medicine, Institute of Chinese Medical Sciences, University of Macau, Macau, People's Republic of China; ${ }^{3}$ Department of Materials Science and Engineering, Stanford University, Stanford, CA, USA

*These authors contributed equally to the work
Correspondence: Hui Li Institute of Chinese Materia Medica, China Academy of Chinese Medical Sciences, I6 Nanxiao Street, Dongzhimen, Dongcheng District, Beijing 100700, People's Republic of China Email lihuiyiren@।63.com

Meiwan Chen

State Key Laboratory of Quality

Research in Chinese Medicine, Institute of Chinese Medical Sciences, University of Macau, Avenue Padre Tomas Pereira SJ, Taipa, Macau 999078, People's Republic of China

Email mwchen@umac.mo
Abstract: Pluronic F127 (F127), an amphiphilic triblock copolymer, has been shown to have significant potential for drug delivery, as it is able to incorporate hydrophobic drugs and selfassemble into nanosize micelles. However, it suffers from dissociation upon dilution owing to the relatively high critical micelle concentration and lack of stimuli-responsive behavior. Here, we synthesized the $\alpha$-tocopherol (TOC) modified F127 polymer (F127-SS-TOC) via a redoxsensitive disulfide bond between F127 and TOC, which formed stable micelles at relatively low critical micelle concentration and was sensitive to the intracellular redox environment. The particle size and zeta potential of the F127-SS-TOC micelles were $51.87 \pm 6.39 \mathrm{~nm}$ and $-8.43 \pm 2.27 \mathrm{mV}$, respectively, and little changes in both particle size and zeta potential were observed within 7 days at room temperature. With $10 \mathrm{mM}$ dithiothreitol stimulation, the F127-SS-TOC micelles rapidly dissociated followed by a significant change in size, which demonstrated a high reduction sensitivity of the micelles. In addition, the micelles showed a high hemocompatibility even at a high micelle concentration $(1,000 \mu \mathrm{g} / \mathrm{mL})$. Low cytotoxicity of the F127-SS-TOC micelles at concentrations ranging from $12.5 \mu \mathrm{g} / \mathrm{mL}$ to $200 \mu \mathrm{g} / \mathrm{mL}$ was also found on both Bel 7402 and L02 cells. Overall, our results demonstrated F127-SS-TOC micelles as a stable and safe aqueous formulation with a considerable potential for drug delivery.

Keywords: Pluronic F127, tocopherol, reduction sensitivity, micelles, hemolysis

\section{Introduction}

Copolymers, containing hydrophilic and hydrophobic portions, are promising multifunctional materials for clinical and biological applications. Owing to their amphiphilic chemical property, with a hydrophilic outer shell and a hydrophobic inner core, they are able to self-assemble into small micellar structures in aqueous solutions with a narrow size distribution of $10-200 \mathrm{~nm} .{ }^{1}$ Because of the small size, nanomicelles tend to sneak into cells, increasing cellular uptake efficiency. Furthermore, nanomicelles also facilitate high tumor accumulation on the basis of enhanced permeability and retention effect. ${ }^{1,2}$ To prove this concept, several polymeric micelles have been developed as drug carriers for cancer chemotherapy..$^{3-5}$ Pluronic F127 (F127, BASF SE, Ludwigshafen, Germany) is one kind of poly(ethylene oxide) (PEO)-poly(propylene oxide) (PPO)-poly(ethylene oxide) (PEO) triblock copolymers, which is accepted as an excipient by the U.S. and British Pharmacopoeia and is widely used in clinical applications. ${ }^{6}$ The amphiphilic chemical structure, including a hydrophobic PPO segment and a hydrophilic PEO segment, allows it to incorporate hydrophobic drugs and self-assemble into micelles with a hydrophilic shell and a hydrophobic core in aqueous medium. ${ }^{7,8}$ Carlson et al $^{9}$ developed the curcumin and resveratrol co-loaded 


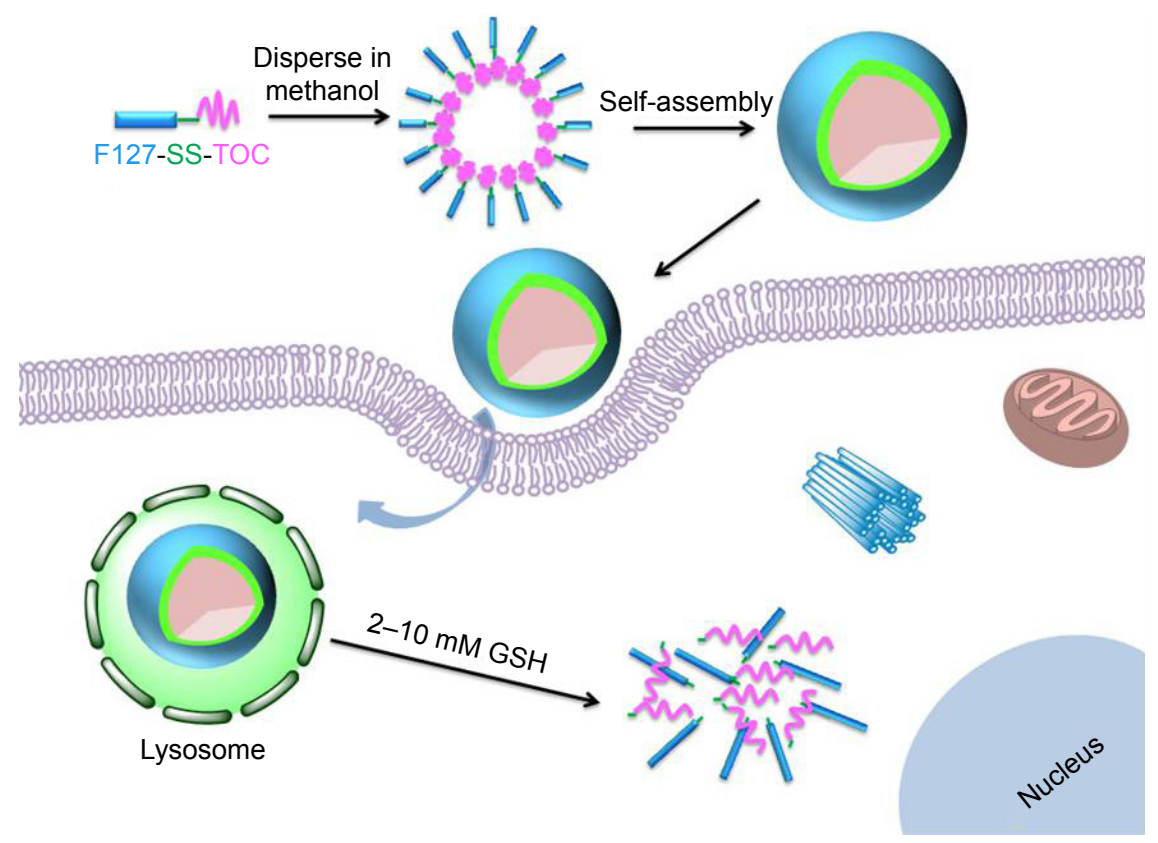

Figure I Schematic illustration of self-assembly of FI27-SS-TOC micelles and hypothetical redox-sensitive micelle rupture in the presence of 2-I0 mM GSH. Abbreviations: FI27, pluronic FI27; FI27-SS-TOC, TOC modified FI27 polymer; GSH, glutathione; TOC, $\alpha$-tocopherol.

micelles based on F127, simultaneously coadministered with doxorubicin hydrochloride, which showed cardioprotective effects and antiproliferative effect in vitro. Although promising, F127 micelles in the blood circulation have one limitation: their relatively high critical micelle concentration (CMC), which prevents them from keeping their structure upon dilution, thereby causing drugs to leak. ${ }^{6,10,11}$

To overcome its limitations and improve the drug delivery functions of F127, several approaches, including chemical modifications or mixed micelles, have been proposed to overcome these disadvantages. ${ }^{12,13}$ Gao et $\mathrm{al}^{6}$ prepared amphiphilic copolymer stearic-coupled F127 nanoparticles to deliver doxorubicin (DOX) for cancer therapy, which had better efficacy compared with free DOX on MCF-7 cells in vitro. Saxena et $\mathrm{al}^{7}$ developed Gambogic acid-loaded Poloxamer 407/D- $\alpha$-Tocopheryl polyethylene glycol 1000 succinate mixed micelles for the treatment of breast and multidrugresistant cancer. $\alpha$-Tocopherol (TOC), known as vitamin E, is toxic to various cancer cells but not to normal cells..$^{14}$ It has a hydrophobic substance consisting of various methylated phenols, making it easy to penetrate into the phospholipid bilayer of the cell membrane. ${ }^{14,15}$ Studies demonstrated that designed functional polymeric micelles were able to stably encapsulate drugs and release them at tumor sites in response to external stimuli such as redox, $\mathrm{pH}$, temperature, and enzyme conditions. ${ }^{16-19}$ In particular, redox-sensitive micelles were designed to trigger burst drug release owing to the high concentration of glutathione ( $\mathrm{GSH}, 4 \mu \mathrm{M} / \mathrm{g}$ ) that is normally found in tumor tissues. ${ }^{20,21}$ Disulfide bond is stable under normal physiological conditions yet responsive to the reductive environment (eg, GSH) of intracellular fluids in cancer cells, indicating that disulfide bonds can be used as the linker of hydrophilic and hydrophobic portions in the redox-sensitive polymeric micelles for controlling the delivery of intracellular drug. ${ }^{22}$

Here, we synthesized a redox-sensitive F127-SS-TOC polymer using a disulfide bond to link F127 and TOC, which formed nanomicellar structures in aqueous solution and ruptured in the presence of glutathione (GSH, 2-10 mM) (Figure 1) owing to a redox reaction. F127-SS-TOC polymer was synthesized and confirmed with ${ }^{1} \mathrm{H}$ nuclear magnetic resonance spectra ( ${ }^{1} \mathrm{H}$ NMR) and Fourier transform infrared spectra (FTIR). The particle size, zeta potential, stability, as well as the reduction sensitivity of the micelles were subsequently evaluated. Then the in vitro hemolysis and cytotoxicity of the F127-SS-TOC micelles were investigated and found to have a high hemocompatibility at a high micelles concentration $(1,000 \mu \mathrm{g} / \mathrm{mL})$ and a low cytotoxicity at concentrations ranging from $12.5 \mu \mathrm{g} / \mathrm{mL}$ to $200 \mu \mathrm{g} / \mathrm{mL}$ on both Bel 7402 and L02 cells. These results demonstrated the potential applications of F127-SS-TOC as stable and safe aqueous formulations for a drug delivery system.

\section{Materials and methods Materials}

Pluronic F127 (F127, molecular weight $=12,600)$ was purchased from BASF SE (Ludwigshafen, Germany). Tocopherol 
and 3,3'-dithiodipropionic acid were purchased from Aladdin Industrial Corporation (Shanghai, People's Republic of China). Dicyclohexylcarbodiimide (DCC) and 4-dimethylaminopyridine (DMAP) were obtained from GL Biochem., Ltd. (Shanghai, People's Republic of China). Millipore water was prepared with a Milli-Q Plus System (Merck Millipore Co., Billerica, MA, USA). All chemicals were in analytical grade.

Human hepatoma cell line Bel 7402 and human hepatic cell line L02 were supplied by American Type Culture Collection (ATCC, Rockville, MD, USA). Dulbecco's Modified Eagle's Medium, fetal bovine serum, phosphatebuffered saline, penicillin-streptomycin, and $0.25 \%(\mathrm{w} / \mathrm{v})$ trypsin/1 mM EDTA were obtained from Thermo Fisher Scientific (Waltham, MA, USA).

\section{Synthesis and characterization of FI27-SS-TOC polymer}

The F127-SS-TOC polymer was synthesized with two-step esterification (Figure 2). ${ }^{23}$ F127-SS was prepared by the conjugation of F127 polymer with 3,3'-dithiodipropionic acid by the esterification reaction. Briefly, F127 polymer (1.0 mmol), 3,3'-dithiodipropionic acid (2.2 mmol), DCC (2.2 mmol), and DMAP (2.2 mmol) were dissolved in $20 \mathrm{~mL}$ anhydrous dimethyl sulphoxide (DMSO) and stirred well for $24 \mathrm{~h}$ at room temperature. Then the resulting solution was dialyzed against DMSO in a dialysis bag with molecular weight cutoff at 3,500 Da to remove unreacted materials, and the by-product $N, N^{\prime}$-dicyclohexylurea was removed by filtration. Next, the dialysis bag was transferred into Millipore water to remove DMSO further. The intermediate product F127-SS was obtained by freeze-drying. Subsequently, TOC was conjugated with F127-SS via the esterification reaction between the carboxyl group of F127-SS and the hydroxy group of TOC. Similarly, F127-SS (1.0 mmol), TOC (2.2 mmol), DCC (2.2 mmol), and DMAP (2.2 mmol) were dissolved in $20 \mathrm{~mL}$ DMSO with stirring for $24 \mathrm{~h}$ at room temperature. The excess of TOC, DCC, and DMAP was removed by dialysis in a dialysis bag (molecular weight
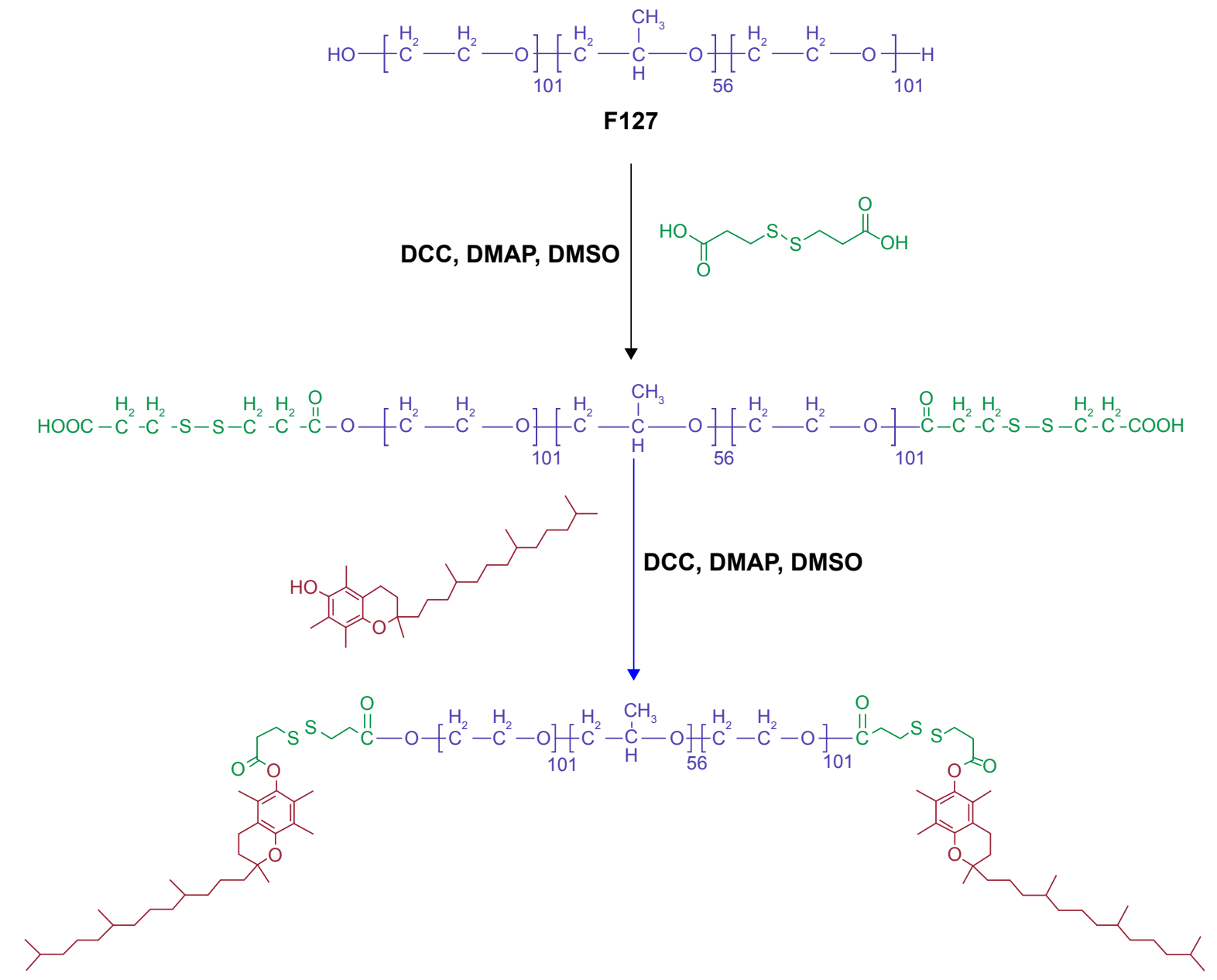

Figure 2 Synthesis route of FI27-SS-TOC polymer.

Abbreviations: DCC, dicyclohexylcarbodiimide; DMAP, 4-dimethylaminopyridine; DMSO, dimethyl sulphoxide; FI27, pluronic FI27; FI27-SS-TOC, TOC modified FI27 polymer; TOC, $\alpha$-tocopherol. 
cutoff $=3,500)$, and by-product $N, N^{\prime}$-dicyclohexylurea was removed by filtration. F127-SS-TOC polymer was obtained by lyophilization and stored at $4{ }^{\circ} \mathrm{C}$ for use.

The structure of F127, F127-SS, TOC, and F127-SS-TOC polymer was measured and confirmed by ${ }^{1} \mathrm{H}$ NMR (Bruker, Karlsruhe, Germany) at $400 \mathrm{MHz}$ in deuterated dimethyl sulfoxide-d6 (DMSO-d6) solvent and FTIR (NEXUS 670, Nicolet, Hudson, NH, USA) within a spectrum range of $400-4,000 \mathrm{~cm}^{-1}$.

\section{Preparation and characterization of FI27-SS-TOC micelles}

F127-SS-TOC micelles were prepared by self-assembly using the solvent evaporation method. ${ }^{24} 15 \mathrm{mg}$ F127-SS-TOC polymer was completely dissolved in $1 \mathrm{~mL}$ methanol and added dropwise into distilled water and stirred well for $6 \mathrm{~h}$ at room temperature. The residual organic solvent was then removed by vacuum evaporation. The final F127-SS-TOC micelles were obtained by filtering with $0.45 \mu \mathrm{m}$ microfiltration membrane and preserved at $4^{\circ} \mathrm{C}$ for use.

Dynamic light scattering (DLS) was employed to measure the average particle size and zeta potential of F127-SS-TOC micelles at room temperature with a Zetasizer Nano ZSP system (Malvern Instruments, Malvern, UK). Micelle morphology was observed and photographed with transmission electron microscopy (TEM, Tecnai G20, FEI Company, Hillsboro, OR, USA). A droplet of micelle sample was placed on the carbon-coated copper grid, followed by staining with phosphotungstic acid solution $(2 \%, \mathrm{w} / \mathrm{v})$. TEM images of the micelles were taken under $200 \mathrm{kV}$.

\section{The stability of FI27-SS-TOC micelles}

The stability of F127-SS-TOC micelles was characterized by measuring the particle size and zeta potential for 7 days. During the whole testing period, the F127-SS-TOC micelles were stored and measured at room temperature with DLS.

Fluorescence spectrometer (Lumina, Thermo Scientific) was applied to measure the CMC of F127-SS-TOC micelles using pyrene as a fluorescence probe. ${ }^{25} \mathrm{~A}$ series of F127-SS-TOC micellar solutions with various concentrations ranging from $1.45 \times 10^{-6} \mathrm{mg} / \mathrm{mL}$ to $0.76 \mathrm{mg} / \mathrm{mL}$ were prepared in the presence of pyrene at $6 \times 10^{-7} \mathrm{M}$. The pyrene fluorescence of different micellar solutions was measured with an excitation wavelength of $338 \mathrm{~nm}$ and an emission wavelength of $380 \mathrm{~nm}$. On formation of micelles, pyrene moved inside the micelles from the aqueous phase, followed by an alteration in the intensity ratio $\mathrm{I}_{338} / \mathrm{I}_{333}$ in a certain micelle concentration range. In addition, a study based on a well-known Tyndall phenomenon was used to further evaluate the stability of the F127-SS-TOC micelles. ${ }^{26}$ In brief, a series of micellar solutions in concentrations ranging from $1.052 \times 10^{-4} \mathrm{mg} / \mathrm{mL}$ to $1.052 \mathrm{mg} / \mathrm{mL}$ were prepared at room temperature and then illuminated with a laser pointer to observe whether the micellar solution showed a visible light beam when irradiated.

\section{The reduction sensitivity of FI27-SS-TOC micelles}

The redox-sensitive behavior of F127-SS-TOC polymer was associated with the size change of micelles in response to dithiothreitol (DTT) (10 mM). $1 \mathrm{~mL}$ DTT (20 mmol/L) was added to $1 \mathrm{~mL}$ F127-SS-TOC micelles to form $10 \mathrm{mM}$ DTT concentration. The solution was incubated in a shaker with a rotation speed of $100 \mathrm{rpm}$ at $37^{\circ} \mathrm{C}$. The reduction-triggered size change of the F127-SS-TOC micelles was traced by DLS at predetermined intervals.

\section{Hemolysis assay}

The hemolytic activity of F127- SS-TOC micelles was carried out in red blood cells from rats following the previous method. ${ }^{27}$ In order to isolate and collect red blood cells (RBCs), fresh rat blood was diluted by physiological saline and centrifuged at $3,000 \mathrm{rpm}$ for $10 \mathrm{~min}$. RBCs were washed several times until the supernatant was clear. After that, the RBCs were diluted with physiological saline to obtain $2 \%$ (v/v) erythrocyte suspension before use. F127-SS-TOC micelles were dissolved in physiological saline at different concentrations, and a suspension of RBCs was added. The mixtures were maintained at $37^{\circ} \mathrm{C}$ for $3 \mathrm{~h}$ in a thermotank, after which they were centrifuged and the supernatant of each sample was collected. The absorbance of supernatant was measured by a microplate reader (SpectraMax M5, Molecular Devices LLC, Sunnyvale, CA, USA) at $540 \mathrm{~nm}$. Distilled water and physiological saline were used as the positive and negative controls, respectively. The hemolysis rate was calculated by the following equation:

$$
\text { Hemolysis rate }(\%)=\frac{A_{\text {sample }}-A_{\text {negative }}}{A_{\text {positive }}-A_{\text {negative }}} \times 100 \text {, }
$$

where $\mathrm{A}_{\text {sample }}, \mathrm{A}_{\text {negative }}$, and $\mathrm{A}_{\text {positive }}$ represented the absorbance of test samples, negative control, and positive control at $540 \mathrm{~nm}$, respectively. All hemolysis experiments were operated in triplicate.

\section{In vitro cytotoxicity study}

The in vitro cytotoxicities of F127-SS-TOC micelles were evaluated on Bel 7402 cells and L02 cells by MTT assay. 
The cells were seeded in 96-well plates at a density of 8,000 cells/well in $100 \mu \mathrm{L}$ Dulbecco's Modified Eagle's Medium containing 10\% fetal bovine serum and incubated at $37^{\circ} \mathrm{C}$ for $24 \mathrm{~h}$. The cells were incubated with F127-SSTOC micelles at concentrations ranging from $12.5 \mu \mathrm{g} / \mathrm{mL}$ to $200 \mu \mathrm{g} / \mathrm{mL}$. After $24-48 \mathrm{~h}$ incubation at $37^{\circ} \mathrm{C}, 20 \mu \mathrm{L}$ of MTT solution ( $5 \mathrm{mg} / \mathrm{mL}$ in phosphate-buffered saline) was added to each well, and the plates were returned to the incubator. The MTT solution was then removed from each well after $4 \mathrm{~h}$. Formazan crystals were dissolved in DMSO. The absorbance of the solution was recorded by a microplate reader at $570 \mathrm{~nm}$. The cell viability (\%) was calculated according to the following equation:

$$
\text { Cell viability }(\%)=\frac{A_{\text {sample }}}{A_{\text {control }}} \times 100
$$

In equation (2), $\mathrm{A}_{\text {sample }}$ and $\mathrm{A}_{\text {control }}$ represented the absorbances of the sample and control wells, respectively, which were measured in triplicate.

\section{Statistical analysis}

The data were presented as mean \pm standard deviation. Each value was the mean of at least three repetitive experiments. Statistical analysis was performed using GraphPad Prism 5 software (GraphPad Software, San Diego, CA, USA).

\section{Results and discussion Synthesis and characterization of FI27-SS-TOC polymer}

F127-SS-TOC polymer was synthesized and confirmed by ${ }^{1} \mathrm{H}$ NMR spectra. As shown in Figure 3, typical signals for F127 polymer were observed, including peaks a $(\delta \sim 1.02 \mathrm{ppm}$ for $\mathrm{CH}_{3}$ of $\mathrm{PPO}$ ), b ( $\delta \sim 3.36 \mathrm{ppm}$ for $\mathrm{CH}$ of $\mathrm{PPO}$ ), and $\mathrm{c}$ ( $\delta=3.41-3.54 \mathrm{ppm}$ for $\mathrm{CH}_{2}$ of PPO and PEO), which appeared in the F127-SS-TOC polymer. In addition, the typical signals of TOC were also found in F127-SS-TOC polymer, including peaks $\mathrm{d}$ ( $\delta=1.95-2.05 \mathrm{ppm}$ for $\mathrm{CH}_{3}$ on the benzene ring), e ( $\delta=1.01-1.15 \mathrm{ppm}$ for $\mathrm{CH}_{2}$ belonged to the repeating units of side chain on TOC polymer), and $\mathrm{f}(\delta=0.75-0.87 \mathrm{ppm}$ for $\mathrm{CH}_{3}$ on the side chain on TOC polymer). The characteristic peaks for $\mathrm{CH}_{2}$ close to the disulfide bond in the F127-SS and F127-SS-TOC polymer were at $2.87 \mathrm{ppm}$ (peak g) and 2.64 ppm (peak h). Moreover, FTIR spectra were used to further validate the structure of intermediate products and F127-SSTOC polymer. Figure 4 showed the FTIR spectra of F127, F127-SS, TOC, and F127-SS-TOC polymer. In the spectra of TOC and F127-SS-TOC, sharp bands at $1,258 \mathrm{~cm}^{-1}$ were attributed to stretching vibration of $-\mathrm{C}-\mathrm{O}$ group. In addition, the bands at 3,485 and 3,475 $\mathrm{cm}^{-1}$ were responsible for stretching vibration of $-\mathrm{OH}$ in $\mathrm{F} 127$ and TOC, respectively. However, the signature peak of hydroxyl did not obviously exist in the F127-SS-TOC polymer, which indicated that this signature peak had disappeared owing to the esterification reaction.

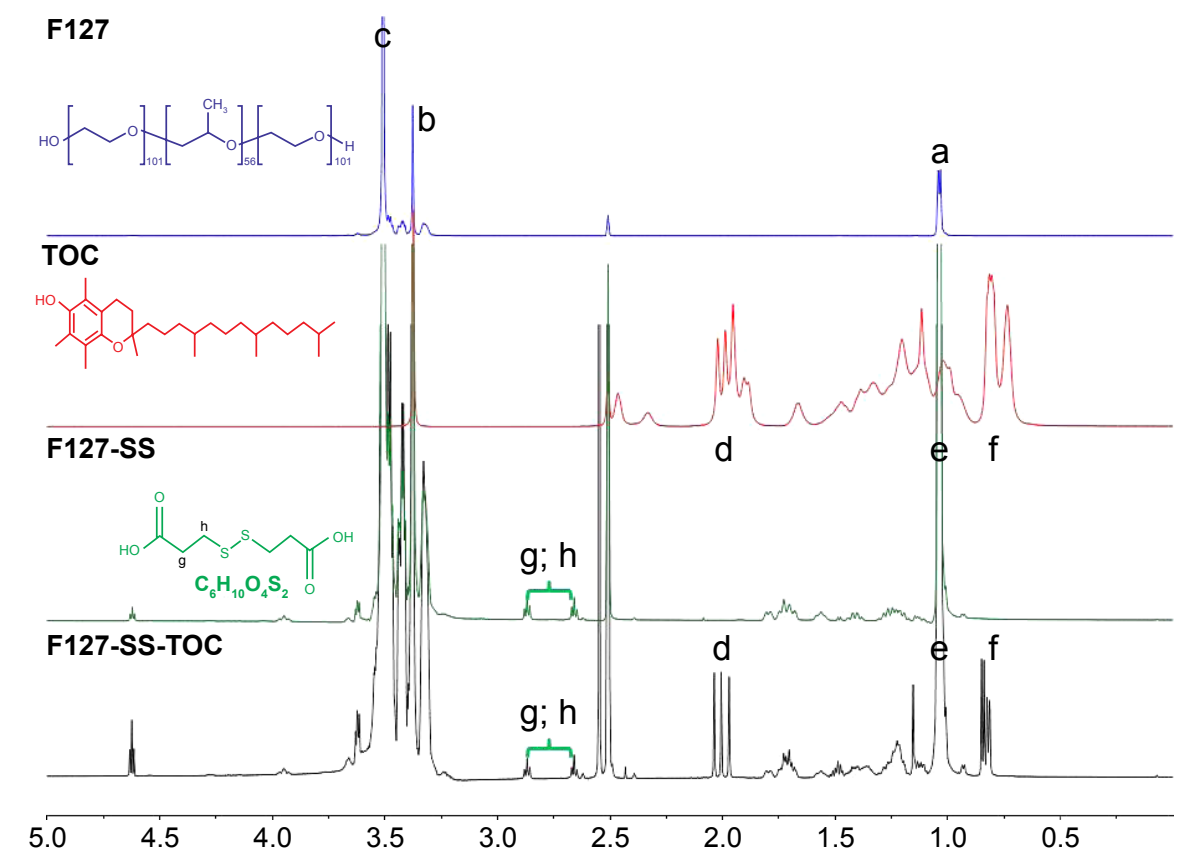

Figure 3 'H NMR spectra of FI27, TOC, FI27-SS, and FI27-SS-TOC polymer.

Note: The letters a-h represent the typical signals in FI27, TOC, FI27-SS, or FI27-SS-TOC polymers.

Abbreviations: FI27, pluronic FI27; FI27-SS, 3,3'-dithiodipropionic acid modified FI27 polymer; FI27-SS-TOC, TOC modified FI27 polymer; 'H NMR, 'H nuclear magnetic resonance spectra; TOC, $\alpha$-tocopherol. 


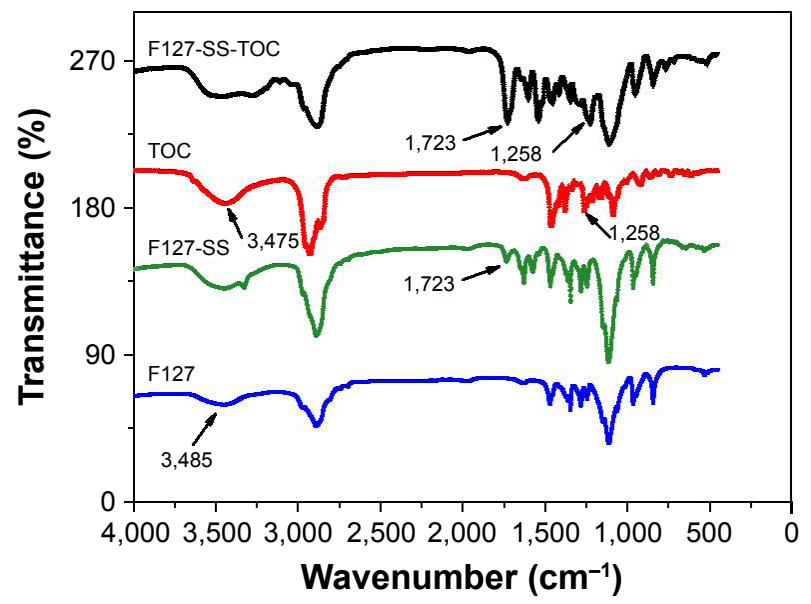

Figure 4 FTIR spectra of FI27, FI27-SS, TOC, and FI27-SS-TOC polymer. Abbreviations: FI27, pluronic FI27; FI27-SS, 3,3'-dithiodipropionic acid modified FI 27 polymer; FI27-SS-TOC, TOC modified FI 27 polymer; FTIR, Fourier transform infrared spectra; TOC, $\alpha$-tocopherol.

Besides, the vibrational peak at $1,723 \mathrm{~cm}^{-1}$ represented the peak of $\mathrm{C}=\mathrm{O}$ in the F127-SS and F127-SS-TOC polymer. Herein, the successful synthesis of the F127-SS-TOC polymer was proved by the ${ }^{1} \mathrm{H}$ NMR and FTIR spectra.

\section{Preparation and characterization of FI27-SS-TOC micelles}

In order to better understand the characteristics of F127SS-TOC micelles, their particle size, zeta potential, and morphology were evaluated. The synthesized F127-SS-TOC polymer was prepared into micelles with a small particle size of 51.87 $\pm 6.39 \mathrm{~nm}$ and narrow distribution (PDI: 0.184 \pm 0.015 ) (Figure 5A), measured by DLS. Furthermore, F127-SS-TOC micelles exhibited negative zeta potential of $-8.43 \pm 2.27 \mathrm{mV}$ (Figure 5B), which might be of benefit to the colloidal stability in aqueous solution. ${ }^{28}$ The morphology of F127SS-TOC micelles (Figure 5C) was observed with TEM, which showed homogeneous spherical shapes and smooth surfaces. However, the particle sizes shown in TEM images were smaller than the results measured by DLS, which was mainly due to the dehydration during the preparation of the TEM samples. ${ }^{29}$ These results indicated that the small particle size of F127-SS-TOC micelles might benefit for tumor-specific accumulation by the enhanced permeability and retention effect.

\section{Stability of FI27-SS-TOC micelles}

Stability is a vital indicator for better understanding of the properties of micelles. As shown in Figure 5D, F127-SS-TOC micelles showed no drastic increases in particle size or zeta potential for 7 days. The average particle sizes were maintained below $100 \mathrm{~nm}$, and the changes in zeta potential were about $\pm 2 \mathrm{mV}$ at room temperature, indicating F127SS-TOC micelles could remain stable during the storage.
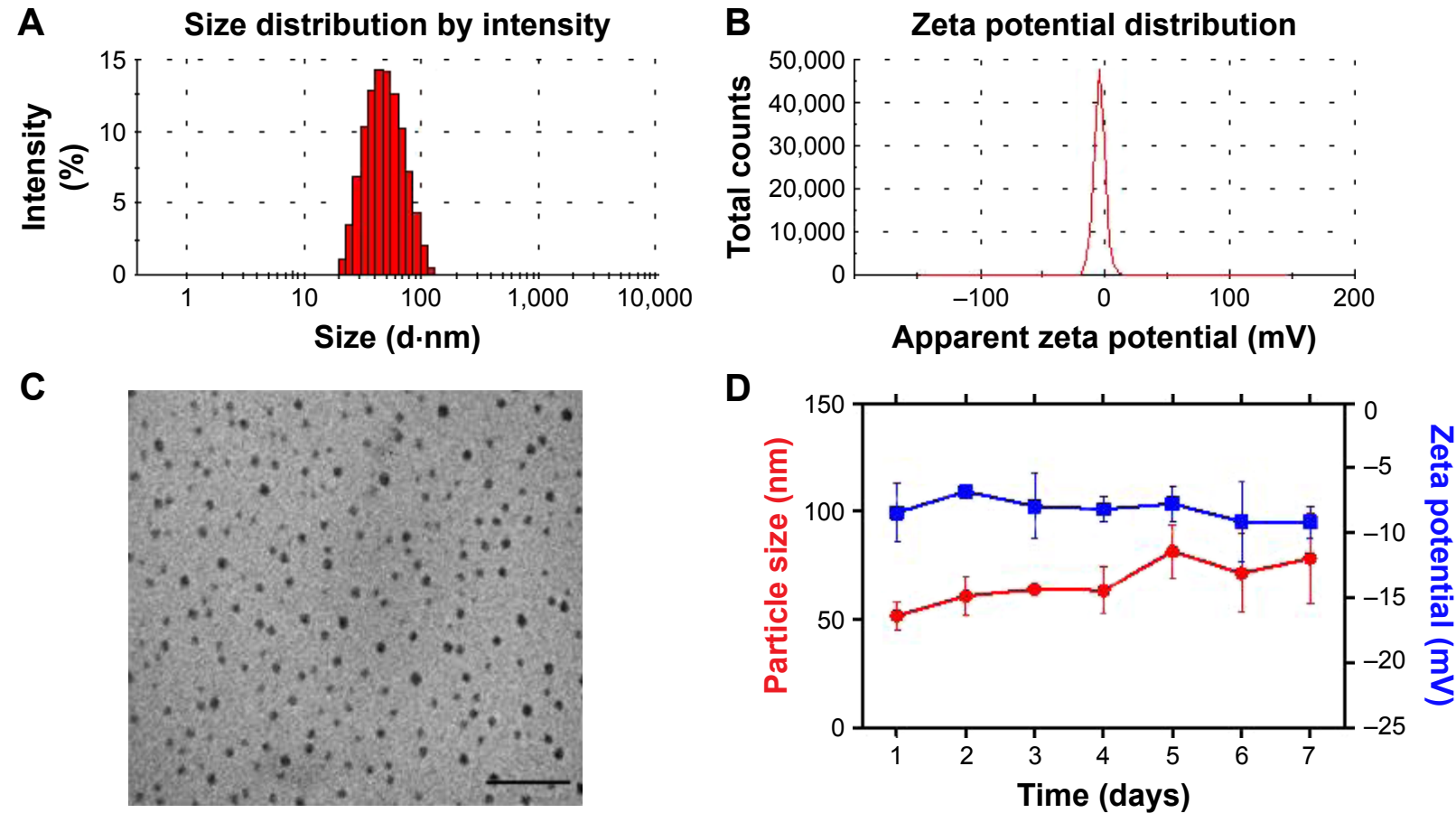

Figure 5 Characterization of FI27-SS-TOC micelles.

Notes: (A) Size distribution of FI27-SS-TOC micelles. (B) Zeta potential of FI27-SS-TOC micelles. (C) TEM images of FI27-SS-TOC micelles. The scale bar corresponds to $200 \mu \mathrm{m}$ in the images. (D) The changes in micellar particle size and zeta potential during 7 days' storage. The red circle represents particle size, and the blue square represents zeta potential. The data were expressed as mean \pm SD of three experiments.

Abbreviations: FI27, pluronic FI27; FI27-SS-TOC, TOC modified FI27 polymer; SD, standard deviation; TEM, transmission electron microscope; TOC, $\alpha$-tocopherol. 
CMC is an important characteristic to evaluate the aggregation properties and reflect the self-assembly stability of F127-SS-TOC micelles. It was determined by pyrene as the fluorescent probe. ${ }^{26,30}$ The CMC of the F127-SS-TOC micelles was $\sim 7.58 \mathrm{mg} / \mathrm{L}\left(\sim 5.4 \times 10^{-7} \mathrm{M}\right)$, which was much lower than the $\mathrm{CMC}\left(6.9 \times 10^{-5} \mathrm{M}\right)$ of Pluronic F127 owing to the conjugation of the hydrophobic TOC chain, ${ }^{11}$ suggesting that F127-SS-TOC micelles could maintain micellization at low concentrations and might have the ability to resist dilution in blood circulation (Figure 6A). Meanwhile, the Tyndall phenomenon of a series of micellar solutions was observed at different concentrations, which were irradiated by a laser beam. ${ }^{26}$ The light beam could penetrate the micellar solutions by irradiation, and the light beam faded with the dilution of the F127-SS-TOC micelles (Figure 6B). The light intensity decreased significantly between the sample S5 $(10.52 \mathrm{mg} / \mathrm{L})$ and $\mathrm{S} 6(5.26 \mathrm{mg} / \mathrm{L})$, and the brightness of sample S6 was similar with water. The low CMC of the F127-SS-TOC micelles could maintain the structural integrity under diluted conditions, which matched the results of the Tyndall phenomenon.

\section{Reduction sensitivity of FI27-SS-TOC micelles}

The reduction-sensitive behavior of the micelles was related to the particle size changes in response to the reducing agent
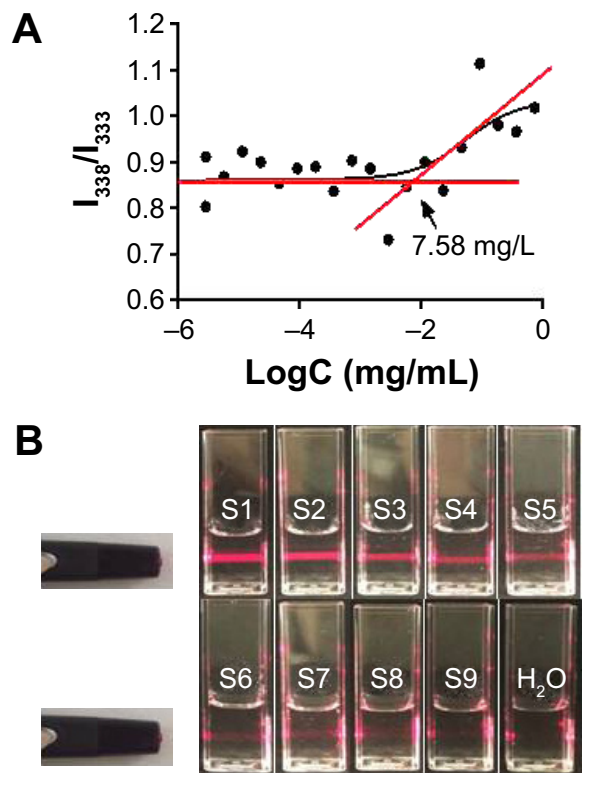

Figure 6 The determination of CMC and Tyndall phenomenon.

Notes: (A) The CMC of the FI27-SS-TOC micelles. The arrow shows that the CMC value of the FI27-SS-TOC micelles was $7.58 \mathrm{mg} / \mathrm{mL}$. (B) Photos of FI27-SSTOC micellar solutions with the irradiation of red laser at various concentrations. (SI: $1.052 \mathrm{mg} / \mathrm{mL}$; S2: $5.260 \times 10^{-1} \mathrm{mg} / \mathrm{mL}$; S3: $1.052 \times 10^{-1} \mathrm{mg} / \mathrm{mL} ; \mathrm{S} 4: 5.260 \times 10^{-2}$ $\mathrm{mg} / \mathrm{mL}$; S5: I.052 $\times 10^{-2} \mathrm{mg} / \mathrm{mL}$; S6: $5.260 \times 10^{-3} \mathrm{mg} / \mathrm{mL} ; \mathrm{S} 7: 1.052 \times 10^{-3} \mathrm{mg} / \mathrm{mL} ; \mathrm{S} 8$ : $5.260 \times 10^{-4} \mathrm{mg} / \mathrm{mL}$, and S9: $1.052 \times 10^{-4} \mathrm{mg} / \mathrm{mL}$ ).

Abbreviations: FI27, pluronic FI27; FI27-SS-TOC, TOC modified FI 27 polymer CMC, critical micelle concentration; LogC, log concentration; TOC, $\alpha$-tocopherol.
DTT (10 mM), measured by DLS. There was no obvious change in the size of the F127-SS-TOC micelles within $3 \mathrm{~h}$ compared with the $0 \mathrm{mM}$ DTT (Figure 7A). Notably, the intensity of the micelles had multiple peaks starting from $5 \mathrm{~h}$, which suggested that the intermediate disulfide bonds between F127 and TOC were cleaved after interaction with $10 \mathrm{mM}$ DTT, resulting in the size changes or eventually inducing aggregation or breakdown of the micelles. In addition, the changes in particle sizes were also verified by TEM. As shown in Figure 7B, $7 \mathrm{~h}$ later, the micelles had irregular surfaces and began to disassemble in the presence of $10 \mathrm{mM}$ DTT, indicating that the F127-SS-TOC micelles were sensitive to reductive conditions.

\section{Hemolysis assay}

Hemolysis is considered to be an essential and reliable measurement to evaluate hemocompatibility of materials. The hemolysis assay was performed according to the previous report, employing rat red blood cells. ${ }^{27}$ The hemolytic activity in vitro of the F127-SS-TOC micelles was evaluated at concentrations of $50,100,500$, and $1,000 \mu \mathrm{g} / \mathrm{mL}$. The F127-SS-TOC micelles did not exhibit hemolysis even at a high concentration of $1,000 \mu \mathrm{g} / \mathrm{mL}$ (Figure 8A). The percentage of hemolysis of the F127-SS-TOC micelles at

A


B

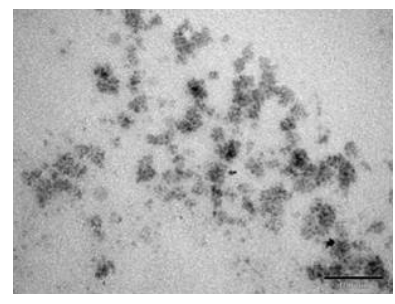

Figure 7 Reduction sensitivity of FI27-SS-TOC micelles in vitro. Notes: (A) Size change with the addition of $10 \mathrm{mM}$ DTT during $7 \mathrm{~h}$ determined by DLS measurement. (B) TEM images of the FI27-SS-TOC micelles with the addition of $10 \mathrm{mM}$ DTT after $7 \mathrm{~h}$. The scale bar corresponds to $100 \mu \mathrm{m}$ in the images. Abbreviations: FI27, pluronic FI27; FI27-SS-TOC, TOC modified FI 27 polymer; DLS, dynamic light scattering; DTT, dithiothreitol; TEM, transmission electron microscope; TOC, $\alpha$-tocopherol. 
A

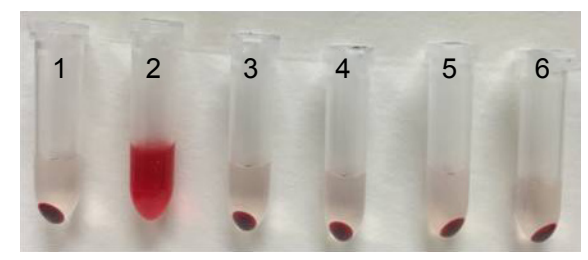

B

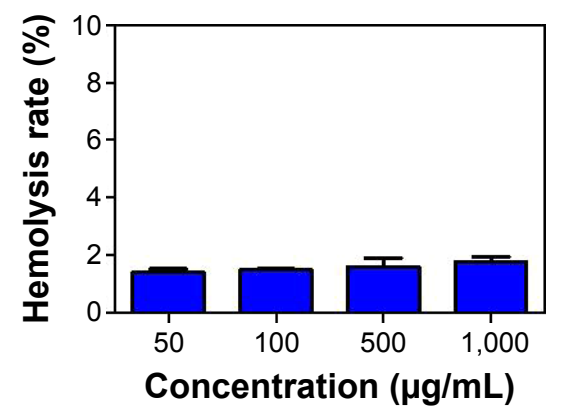

Figure 8 The hemolysis assay of FI27-SS-TOC micelles.

Notes: (A) RBC suspensions were centrifuged after incubation with FI27-SSTOC micelles of various concentrations for $3 \mathrm{~h}$. Sample I represented the negative control (RBCs suspended in physiological saline, 0\% lysis); Sample 2 represented positive control (RBCs suspended in distilled water, 100\% lysis); Samples 3, 4, 5, and 6 represented the RBCs suspended in $50,100,500$, and $\mathrm{I}, 000 \mu \mathrm{g} / \mathrm{mL}$ concentrations of FI27-SS-TOC micelles, respectively. (B) The hemolysis rate (\%) of RBCs incubated with FI27-SS-TOC micelles at various concentrations. The data were expressed as mean \pm SD of three experiments.

Abbreviations: $\mathrm{FI} 27$, pluronic $\mathrm{FI} 27$; FI27-SS-TOC, TOC modified FI 27 polymer; RBC, red blood cell; SD, standard deviation; TOC, $\alpha$-tocopherol.

different concentrations was calculated using equation (1). As shown in Figure 8B and Table 1, hemolysis rates of the F127-SS-TOC micelles at concentrations of 50, 100, 500, and $1,000 \mu \mathrm{g} / \mathrm{mL}$ were $1.387 \% \pm 0.122 \%, 1.438 \% \pm 0.042 \%$, $1.589 \% \pm 0.297 \%$, and $1.745 \% \pm 0.205 \%$, respectively, which showed similar hemolysis rates with no statistically significant difference among different concentrations. In accordance with the American Society for Testing and Materials (ASTM F756-00, 2000), the materials were classified, according to the degree of hemolysis, into three kinds: hemolytic ( $>5 \%$ of hemolysis), slightly hemolytic $(2 \%-5 \%$ of hemolysis), and nonhemolytic ( $0 \%-2 \%$ of hemolysis). ${ }^{31}$ The hemolysis rates for all four concentrations were $<2 \%$, which demonstrated the excellent blood compatibility of the F127-SS-TOC micelles as well as their potential application as safe carriers for intravenous injection.

Table I Quantification of hemolysis test of FI27-SS-TOC micelles with different concentrations (mean $\pm \mathrm{SD}, \mathrm{n}=3$ )

\begin{tabular}{lllll}
\hline FI27-SS-TOC & 1,000 & 500 & 100 & 50 \\
$\begin{array}{l}\text { concentration } \\
(\mu \mathrm{g} / \mathrm{mL})\end{array}$ & & & & \\
Hemolysis rate $(\%)$ & $1.745 \pm 0.205$ & $1.589 \pm 0.297$ & $1.483 \pm 0.042$ & $1.387 \pm 0.122$ \\
\hline
\end{tabular}

Abbreviations: FI27, pluronic FI27; FI27-SS-TOC, TOC modified FI27 polymer; $\mathrm{n}$, number; SD, standard deviation; TOC, $\alpha$-tocopherol.
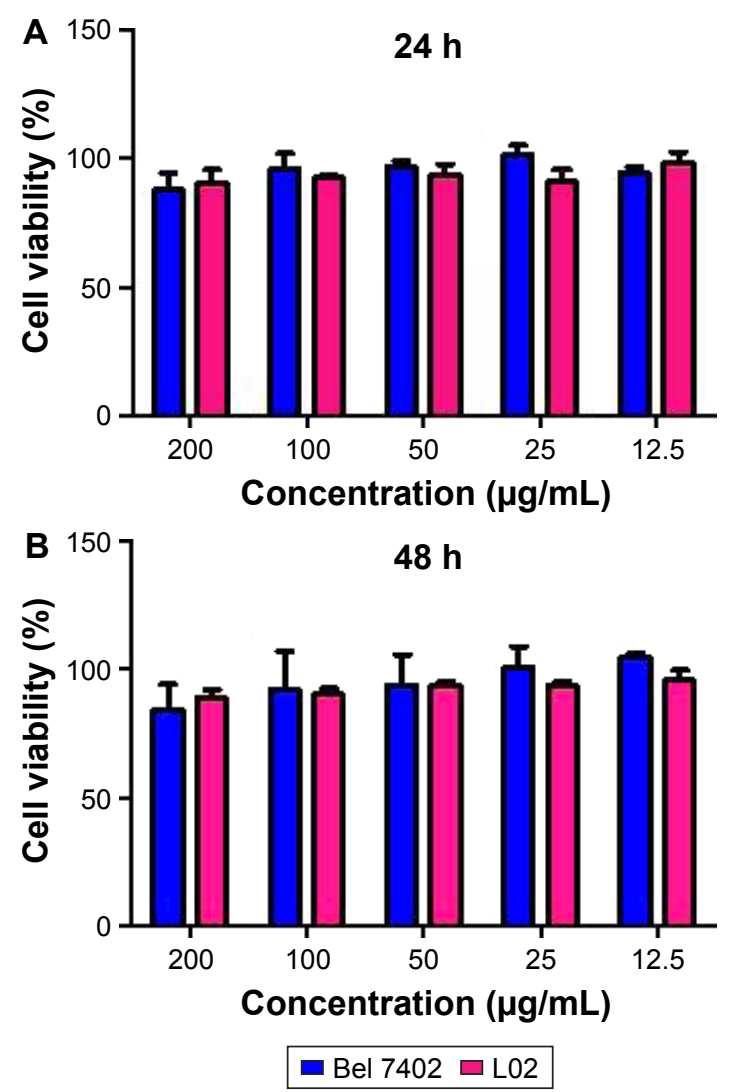

Figure 9 Cell viability of Bel 7402 and L02 cells treated with different concentrations of FI27-SS-TOC micelles for (A) $24 \mathrm{~h}$ and (B) $48 \mathrm{~h}$.

Note: The data were expressed as mean \pm SD of three experiments.

Abbreviations: $\mathrm{FI} 27$, pluronic $\mathrm{FI} 27$; FI27-SS-TOC, TOC modified FI 27 polymer; SD, standard deviation; TOC, $\alpha$-tocopherol.

\section{In vitro cytotoxicity}

The cytotoxicity of polymeric micelles is a crucial parameter for medical application. The cytotoxicity of the F127SS-TOC against the Bel 7402 and L02 cells was evaluated by the MTT assay in terms of cell viability. ${ }^{15}$ The Bel 7402 and L02 cells were treated with various concentrations of F127-SS-TOC micelles for $24 \mathrm{~h}$ and $48 \mathrm{~h}$, respectively. As shown in Figure 9, the F127-SS-TOC micelles did not show obvious cytotoxicity, with the micellar concentrations ranging from 12.5 to $200 \mu \mathrm{g} / \mathrm{mL}$. After incubation for $24 \mathrm{~h}$, the cell viability of both cells was $>85 \%$. Even after incubation for $48 \mathrm{~h}$, the F127-SS-TOC micelles showed low cytotoxicity with a cell viability of about $85 \%$ in vitro. These results demonstrated the low biological toxicity of the F127-SSTOC micelles at certain concentrations and its potential in the biomedical field.

\section{Conclusion}

In summary, we developed a biodegradable disulfidecontaining polymer F127-SS-TOC, which proved to have 
redox-sensitive properties in vitro. The micelles based on the F127-SS-TOC polymer had homogeneous spherical shapes and smooth surfaces, with a particle size as small as $51.87 \pm 6.39 \mathrm{~nm}$. The zeta potential of the micelles was $-8.43 \pm 2.27 \mathrm{mV}$, indicating that it might be beneficial for the colloidal stability in aqueous solution. Besides, the F127-SS-TOC micelles were proved to be stable at room temperature within 7 days, and the low CMC could ensure that the micelles maintained self-assembly under diluted conditions. Furthermore, the obvious reduction sensitivity of the F127-SS-TOC micelles was verified by measuring the size change in the presence of $10 \mathrm{mM}$ DTT. The F127-SSTOC micelles possessed favorable blood compatibility even at a micelle concentration of $1,000 \mu \mathrm{g} / \mathrm{mL}$. In vitro study showed low cytotoxicity of the F127-SS-TOC micelles, indicating that the F127-SS-TOC polymer is safe to use. Thus, the F127-SS-TOC polymer exhibits excellent properties as micelles and has the potential to become a redoxsensitive carrier for delivering hydrophobic agents in cancer therapy. Besides, we will fully elucidate the application of drug-loaded micelles based on the F127-SS-TOC polymer in further work.

\section{Acknowledgments}

This study was supported by the Macao Science and Technology Development Fund (096/2015/A3), the Research Fund of the University of Macau (MYRG2014-00033ICMS-QRCM, MYRG2014-00051-ICMS-QRCM, MYRG2015-00171-ICMS-QRCM), and the National Natural Science Foundation of China (81403120). This study was also supported by Beijing Joint Project Specific Funds, Fund of State Administration of TCM (200907001-5), the Support Program of the Institute of Chinese Materia Medica, China Academy of Chinese Medical Sciences (Z02063, Z184), and the Innovative Funding for PhD Students at China Academy of Chinese Medical Sciences (CX201606).

\section{Disclosure}

The authors report no conflicts of interest in this work.

\section{References}

1. Arshad M, Saied S, Ullah A. PEG-lipid telechelics incorporating fatty acids from canola oil: synthesis, characterization and solution selfassembly. Rsc Adv. 2014;4(50):26439-26446.

2. Biswas S, Kumari P, Lakhani PM, Ghosh B. Recent advances in polymeric micelles for anti-cancer drug delivery. Eur J Pharm Sci. 2016;83: 184-202.

3. Krishnamurthy S, Ng VW, Gao S, Tan MH, Yang YY. Phenformin-loaded polymeric micelles for targeting both cancer cells and cancer stem cells in vitro and in vivo. Biomaterials. 2014;35(33):9177-9186.
4. Ke XY, Lin Ng VW, Gao SJ, Tong YW, Hedrick JL, Yang YY. Co-delivery of thioridazine and doxorubicin using polymeric micelles for targeting both cancer cells and cancer stem cells. Biomaterials. 2014;35(3):1096-1108.

5. Shahin M, Ahmed S, Kaur K, Lavasanifar A. Decoration of polymeric micelles with cancer-specific peptide ligands for active targeting of paclitaxel. Biomaterials. 2011;32(22):5123-5133.

6. Gao Q, Liang Q, Yu F, Xu J, Zhao Q, Sun B. Synthesis and characterization of novel amphiphilic copolymer stearic acid-coupled F127 nanoparticles for nano-technology based drug delivery system. Colloids Surf B Biointerfaces. 2011;88(2):741-748.

7. Saxena V, Hussain MD. Poloxamer 407/TPGS mixed micelles for delivery of gambogic acid to breast and multidrug-resistant cancer. Int J Nanomedicine. 2012;7:713-721.

8. Dehghan Kelishady P, Saadat E, Ravar F, Akbari H, Dorkoosh F. Pluronic F127 polymeric micelles for co-delivery of paclitaxel and lapatinib against metastatic breast cancer: preparation, optimization and in vitro evaluation. Pharm Dev Technol. 2015;20(8):1009-1017.

9. Carlson LJ, Cote B, Alani AW, Rao DA. Polymeric micellar co-delivery of resveratrol and curcumin to mitigate in vitro doxorubicin-induced cardiotoxicity. J Pharm Sci. 2014;103(8):2315-2322.

10. Gao N, Lu S, Gao C, et al. Injectable shell-crosslinked F127 micelle/ hydrogel composites with $\mathrm{pH}$ and redox sensitivity for combined release of anticancer drugs. Chem Eng J. 2016;287:20-29.

11. Sezgin Z, Yuksel N, Baykara T. Preparation and characterization of polymeric micelles for solubilization of poorly soluble anticancer drugs. Eur J Pharm Biopharm. 2006;64(3):261-268.

12. Chen L, Sha X, Jiang X, Chen Y, Ren Q, Fang X. Pluronic P105/F127 mixed micelles for the delivery of docetaxel against taxol-resistant non-small cell lung cancer: optimization and in vitro, in vivo evaluation. Int J Nanomedicine. 2013;8:73-84.

13. Guo DD, Moon HS, Arote R, et al. Enhanced anticancer effect of conjugated linoleic acid by conjugation with Pluronic F127 on MCF-7 breast cancer cells. Cancer Lett. 2007;254(2):244-254.

14. Nam JP, Lee KJ, Choi JW, Yun CO, Nah JW. Targeting delivery of tocopherol and doxorubicin grafted-chitosan polymeric micelles for cancer therapy: in vitro and in vivo evaluation. Colloids Surf B Biointerfaces. 2015;133:254-262.

15. Fang X, Xu Y, Zhang J, Lu X, Wang Y, Chen M. Synthesis and characterization of an amphiphilic linoleic acid-g-quaternary chitosan with low toxicity. J Nanomaterials. 2015;(16)1:1-7.

16. Chen W, Zhong P, Meng F, et al. Redox and pH-responsive degradable micelles for dually activated intracellular anticancer drug release. J Control Release. 2013;169(3):171-179.

17. Harnoy AJ, Rosenbaum I, Tirosh E, et al. Enzyme-responsive amphiphilic PEG-dendron hybrids and their assembly into smart micellar nanocarriers. J Am Chem Soc. 2014;136(21):7531-7534.

18. Chen YC, Liao LC, Lu PL, et al. The accumulation of dual $\mathrm{pH}$ and temperature responsive micelles in tumors. Biomaterials. 2012;33(18): 4576-4588.

19. Hu YW, Du YZ, Liu N, et al. Selective redox-responsive drug release in tumor cells mediated by chitosan based glycolipid-like nanocarrier. J Control Release. 2015;206:91-100.

20. Deng B, Ma P, Xie Y. Reduction-sensitive polymeric nanocarriers in cancer therapy: a comprehensive review. Nanoscale. 2015;7(30):12773-12795.

21. Kuppusamy P, Li H, Ilangovan G, et al. Noninvasive imaging of tumor redox status and its modification by tissue glutathione levels. Cancer Res. 2002;62(1):307-312.

22. Wu B, Yu P, Cui C, et al. Folate-containing reduction-sensitive lipidpolymer hybrid nanoparticles for targeted delivery of doxorubicin. Biomater Sci. 2015;3(4):655-664.

23. Fang XB, Zhang JM, Xie X, et al. pH-sensitive micelles based on acid-labile pluronic F68-curcumin conjugates for improved tumor intracellular drug delivery. Int J Pharm. 2016;502(1-2):28-37.

24. Huang S, Yu X, Yang L, et al. The efficacy of nimodipine drug delivery using mPEG-PLA micelles and mPEG-PLA/TPGS mixed micelles. Eur J Pharm Sci. 2014;63:187-198. 
25. Bao Y, Guo Y, Zhuang X, et al. D-alpha-tocopherol polyethylene glycol succinate-based redox-sensitive paclitaxel prodrug for overcoming multidrug resistance in cancer cells. Mol Pharm. 2014;11(9):3196-3209.

26. Lv S, Tang Z, Zhang D, et al. Well-defined polymer-drug conjugate engineered with redox and $\mathrm{pH}$-sensitive release mechanism for efficient delivery of paclitaxel. J Control Release. 2014;194:220-227.

27. Liu S, Huang W, Jin MJ, Fan B, Xia GM, Gao ZG. Inhibition of murine breast cancer growth and metastasis by survivin-targeted siRNA using disulfide cross-linked linear PEI. Eur J Pharm Sci. 2016;82:171-182.

28. Duan X, Xiao J, Yin Q, et al. Smart pH-sensitive and temporalcontrolled polymeric micelles for effective combination therapy of doxorubicin and disulfiram. Acs Nano. 2013;7(7):5858-5869.
29. Fang X, Xu Y, Wang S, Wan J, He C, Chen M. Pluronic F68-linoleic acid nano-spheres mediated delivery of gambogic acid for cancer therapy. AAPS PharmSciTech. 2017;18(1):147-155.

30. Cai Y, Wang S, Wu M, et al. PCL-F68-PCL/PLGA-PEG-PLGA mixed micelles mediated delivery of mitoxantrone for reversing multidrug resistant in breast cancer. Rsc Adv. 2016;6(42):35318-35327.

31. Andrade FK, Silva JP, Carvalho M, Castanheira EM, Soares R, Gama M. Studies on the hemocompatibility of bacterial cellulose. J Biomed Mater Res A. 2011;98(4):554-566.

\section{Publish your work in this journal}

The International Journal of Nanomedicine is an international, peerreviewed journal focusing on the application of nanotechnology in diagnostics, therapeutics, and drug delivery systems throughout the biomedical field. This journal is indexed on PubMed Central, MedLine, CAS, SciSearch ${ }^{\circledR}$, Current Contents ${ }^{\circledR} /$ Clinical Medicine,
Journal Citation Reports/Science Edition, EMBase, Scopus and the Elsevier Bibliographic databases. The manuscript management system is completely online and includes a very quick and fair peer-review system, which is all easy to use. Visit http://www.dovepress.com/ testimonials.php to read real quotes from published authors. 